\title{
El PAPEL DE LOS Sistemas de INFORMACIÓN EN LA GESTIÓN DEL CONOCIMIENTO ORGANIZACIONAL
}

\section{Lorena Litai Ramos Luna}

Lucia Lizbeth Morales Herrera

\section{José R. Morales Calderón ${ }^{1}$}

\section{Resumen}

El conocimiento se ha convertido un elemento central para el desarrollo económico de las organizaciones, por lo que se ha vuelto susceptible de ser gestionado mediante Sistemas de Información, los cuales fungen como herramientas que posibilitan su almacenaje, procesamiento y distribución en distintos niveles. En este entendido, el objetivo de este documento es analizar a los sistemas de información en su carácter técnico, pero también social, desde el papel que juegan en la gestión del conocimiento organizacional a partir del modelo de Nonaka y Takeuchi (1995). Para cumplir este propósito, el documento se ha dividido en dos apartados. En el primero, se describe de manera general el desarrollo, funcionamiento, proceso de construcción y finalidad de los sistemas de información. En el segundo, se define la gestión del conocimiento y se detallan las formas de conversión del conocimiento de Nonaka y Takeuchi (1995) incorporando en el análisis a los sistemas de información. Por último, se concluye que cada organización crea sus propias estrategias, estructuras y formas de gestionar el conocimiento a partir de los sistemas de información.

Palabras clave: Estrategia, toma de decisiones, cultura, aprendizaje organizacional.

1 Profesores del Departamento de Economía de la Universidad Autónoma Metropolitana, Unidad Iztapalapa. 


\section{Abstract}

Knowledge has become a central element for the economic development of organizations, so it has become susceptible to be managed through Information Systems, which act as tools that enable their storage, processing and distribution at different levels. In this understanding, the objective of this document is to analyze information systems in their technical, but also social, from the role they play in the management of organizational knowledge from the model of Nonaka and Takeuchi (1995). To fulfill this purpose, the document has been divided into two sections. In the first one, the development, operation, construction process and purpose of the information systems are described in a general way. In the second, knowledge management is defined and the knowledge conversion forms of Nonaka and Takeuchi (1995) are detailed, incorporating information systems into the analysis. Finally, it is concluded that each organization creates its own strategies, structures and ways of managing knowledge from information systems.

Keywords: Strategy, decision making, culture, organizational learning.

\section{Introducción}

Las organizaciones en la sociedad de la información y el conocimiento requieren de la movilización de sus activos intangibles, es decir aquellos recursos que no se pueden medir cuantitativamente pero que agregan valor cualitativo a la entidad a la que pertenecen, se consideran tácitos y aumentan valor al volverse rutinarios (Gil, López, Molina y Bolio, 2011; Lovera, 2009; Rivera, 2006; Sveiby, 1997). El resultado de tal movilización deriva en la innovación (López, 2002; Mazzotti, Gonzáles y Solís, 2011). La herramienta principal en dicha tarea se encuentra en los Sistemas de Información (SI), los cuales son instrumentos para el manejo de la información (Silva, 2006).

Para contextualizar este hecho, téngase en cuenta que la sociedad de la información es el término que utiliza Castells (2000) al denominar la productividad y competitividad de los agentes económicos, en función 
de su capacidad para producir, procesar y aplicar de manera eficaz la información basada en el conocimiento. Es un fenómeno a nivel global en el que interactúan individuos, grupos y organizaciones en una estructura de red. El avance tecnológico propició la base material de su constitución, pues implica una transformación en el modo de procesar la información producto de la actividad humana.

Ahora bien, la sociedad del conocimiento hace evidente la centralidad de la producción y distribución del conocimiento en la dinámica y el desarrollo económico; el conocimiento, al tener un amplio valor en el mercado, se sitúa en las organizaciones como un activo inagotable que genera éxito y perpetuidad, y es susceptible de ser gestionado. Dicha gestión se apoya en las Tecnologías de la Información y la Comunicación (TIC), las cuales tienen preponderancia en la dinámica del sistema porque permiten almacenar grandes volúmenes de conocimiento o información y transferirlos, posibilitando la interacción entre los miembros de la organización o de varias organizaciones sin importar ni el tiempo ni el lugar (Morales, 2012; Vessuri, 2008).

La sociedad de la información y del conocimiento ha generado nuevas formas de trabajo y productividad de la mano de obra. En este entendido, la fuente de extracción de los conocimientos en las organizaciones son los individuos ${ }^{2}$ que no poseen capital propio, pero sí habilidades para poner en práctica en espacios productivos, sociales e institucionales (Mazzotti, Gonzáles y Solís, 2011); de ahí que exista la primacía del desarrollo del capital humano mediante la capacitación, entrenamiento y educación (Didriksson, 2015; 2016). En las organizaciones, los sistemas de la información son útiles al almacenar el conocimiento extraído susceptible de ser gestionado.

Tomando en cuenta que los sistemas de la información se han convertido en un componente estratégico para las organizaciones y es fundamental en la toma de decisiones e, incluso considerado como ventaja

2 En términos de Nonaka (2007) a estos sujetos puede nombrárseles trabajadores del conocimiento, emprendedores de los que surge nuevo conocimiento valioso para las organizaciones. 
competitiva ${ }^{3}$ (Andrade, 2003; Abrego, Sánchez y Medina, 2016; López, 2002; Lovera, 2009; Rivera, 2006; Silva, 2006), el presente trabajo propone analizar a los sistemas de la información, en su carácter técnico, pero también social, desde el papel que juegan en la gestión del conocimiento organizacional a partir del modelo de Nonaka y Takeuchi (1995). Para ello, el desarrollo se divide en dos apartados. En el primero, se describe de manera general el desarrollo, funcionamiento, proceso de construcción y finalidad de los sistemas de información. En el segundo, se define la gestión del conocimiento y se detallan las formas de conversión del conocimiento de Nonaka y Takeuchi (1995) incorporando en el análisis a los SI.

\section{Los sistemas de información}

A principios de la década de los sesenta, el papel de los sistemas de la información se centraba únicamente en el procesamiento de transacciones, mantenimiento de registros contables y otras aplicaciones de procesamiento de datos. Más adelante su utilización se extendió hacia suministrar información a los usuarios finales, es decir los gerentes, para auxiliarlos en la toma de decisiones, de modo que les permitiera enfrentar problemas específicos. En la década de los ochenta, el nuevo rol se vinculó con la aparición de paquetes de software y redes de telecomunicaciones que se sustentaron en el uso de computadoras para apoyar los procesos de trabajo. La información se diseminó entonces a lo largo de las organizaciones, desarrollándose el concepto de Sistemas de Información, una manera fácil de obtener información crítica. Durante la década de los noventa, con el crecimiento del internet e intranet, se ampliaron las capacidades de los Si (López, 2002). La propuesta que se desarrolla actualmente tiene que ver con su papel en la gestión del conocimiento.

3 La ventaja competitiva forma parte del marco teórico desarrollado por Porter, en el que se propone un sistema para analizar las ventajas competitivas llamado modelo de la cadena de valor, que es una teoría sistemática que consiste en examinar todas las actividades de una compañía y cómo se relaciona. Crea también el modelo de las cinco fuerzas para entender la estructura de una industria y cómo cambia (Lovera, 2009). Para Nonaka (2007), la única ventaja competitiva, duradera y segura es el conocimiento. 
Son múltiples las definiciones existentes sobre los sistemas de información. Por ejemplo, Abrego, Sánchez y Medina (2016) demarcan que son herramientas capaces de reunir, procesar, distribuir y compartir datos de manera oportuna e integrada. Para Arellano (2008), los SI permiten calcular, comprar, clasificar, ordenar y distribuir información sintetizada. López (2002) por su parte señala que un SI es un conjunto de componentes interrelacionados que permiten capturar, procesar, almacenar, y distribuir la información. Las concepciones anteriores coinciden en que los SI son herramientas que apoyan en la extracción, procesamiento, almacenaje y distribución de la información. No obstante, téngase en cuenta que los sistemas de la información representan también un conjunto de medios humanos y materiales encargados del tratamiento de la información (Abrego, Sánchez y Medina, 2016).

El funcionamiento de los sistemas de la información depende de la entrada de información, almacenamiento, procesamiento y salida. El sistema toma los datos para procesarlos, pudiendo ser alimentado manualmente por los usuarios que lo ocupan en sus actividades, para posteriormente distribuirlos en sistemas o módulos. Es fundamental el elemento técnico, donde se agrupa el hardware y el software que permite su puesta en marcha (López, 2002; Silva, 2006). Su implantación representa una innovación importante a la organización (López, 2002) y la inversión se justifica ante la ola de beneficios que promete en términos de ganancias (Abrego, Sánchez y Medina, 2016).

El proceso de construcción de los sistemas de la información requiere de un análisis de la tarea y la actividad según Kafure (2010). La descripción de la tarea se obtiene por medio de la recolección de datos a partir de instrumentos como la entrevista y la observación para documentar evidencia sobre el proceso de realización. El análisis de la tarea se efectúa por medio de la observación de aspectos críticos en el sitio de trabajo. Una vez hecha la recolección de datos, se elabora un prototipo que crea la interfaz inicial para cotejarla con la participación de un grupo objetivo.

La finalidad que auspician los sistemas de la información va desde la toma de decisiones (Arellano, 2008; López, 2002; Silva, 2006; Andra- 
de, 2003), la canalización de la información (Torres y Lamenta, 2015), el soporte a los usuarios para prestar mejores servicios (Kafure, 2010), el aumento de la productividad (Arellano, 2008), el apoyo en la gestión del conocimiento de la organización (Arellano, 2008), entre otras. Esta última, es el eje central en este documento. En el siguiente apartado, se explica qué es la gestión del conocimiento y con base en las formas de conversión del conocimiento de Nonaka y Takeuchi (1995), se analiza el papel de éste en el proceso.

\section{La gestión del conocimiento}

La gestión del conocimiento es una proceso sistémico y específico cuya utilización se extiende a través de distintas organizaciones (García y Gómez, 2015; Gil et. al., 2011). García y Gómez (2015) delimitan que la incorporación del conocimiento de la empresa en la creación de estrategias generó tal acepción, que se formalizó como un proceso sistemático de buscar y encontrar la información que surge en las organizaciones para ser transformada a través de la cooperación de las personas en un proceso de conocimiento que permita la consecución de los objetivos.

Entre las definiciones existentes señaladas por los autores mencionados, se encuentra la de Nonaka y Takeuchi (1995), quienes delimitan a la gestión del conocimiento como la capacidad de la empresa de crear conocimiento nuevo, diseminarlo e incorporarlo a los procesos de la organización; Sveiby (1997) que lo enuncia como el arte de crear valor a partir de activos intangibles; Tejedor y Aguirre (1998) como el conjunto de procesos que permiten utilizar el conocimiento como factor clave para añadir y generar valor; Moya-Angeler (2011) quienes argumentan que la gestión del conocimiento trata de extraer lo mejor de las personas en la organización utilizando sistemas que permiten que la información disponible se convierta en conocimiento; y finalmente, Lindblom y Tijjannen (2010) lo conciben como un proceso deliberado que busca la obtención del conocimiento de las personas para mejorar la competitividad organizacional. A manera de agrupar tales acepciones, considérese que es un proceso constante, dado que implica una serie de etapas organizadas que se retroalimentan unas de 
otras; dicho proceso busca apropiarse del conocimiento que los individuos generan en la práctica para así, mediante el uso de SI, procesarlo, almacenarlo y distribuirlo de forma estratégica.

La gestión del conocimiento no debe confundirse con la gestión de la información, ésta última es el conjunto de actividades que se realizan con el propósito de identificar, adquirir, organizar y almacenar la información que se produce o recibe en una organización para el desarrollo de sus actividades, en el tiempo y lugar oportuno mediante el uso de TIC's (Álvarez, 2006; Gil et. al., 2011; Mena, 2011). La información es el conjunto de datos simbólicos, verbales y gráficos organizados, dirigidos a los individuos para su análisis, reflexión e interpretación (Gil et. al., 2011; Rivera, 2006). Por otro lado, el conocimiento es originado por los individuos al crear y organizar sus experiencias (Nonaka y Takeuchi, 1995; Polanyi, 1958). Existen dos tipos de conocimiento de acuerdo con Polanyi (1958): el conocimiento tácito y el conocimiento explícito.

El conocimiento tácito es subjetivo y comprende las experiencias de trabajo, habilidades y creencias (Polanyi, 1958). Es personal y se comparte asimilando destrezas mediante la observación, la imitación y la práctica (Nonaka, 2007). Este tipo de conocimiento es la base de la creación de conocimiento organizacional (Nonaka y Takeuchi, 1995). Incluye elementos cognoscitivos y técnicos. Los primeros son modelos mentales como esquemas, perspectivas, creencias y puntos de vista que ayudan a los individuos a percibir y definir su mundo. Por otra parte, el conocimiento explícito es codificado y transmisible a través de algún sistema de lenguaje formal (Polanyi, 1958). Para que el conocimiento tácito se convierta en tecnologías y productos concretos, debe convertirse en conocimiento explícito. Su asimilación se logra cuando pasa de ser explícito a tácito mediante la interacción social (Nonaka, 2007).

Ahora bien, los enfoques sobre la gestión del conocimiento implican elementos tales como el individuo, las tecnologías de la información e incluso las políticas y procedimientos propios de la gestión. Nonaka y Takeuchi (1995), presentan un modelo en el que explican que la innovación en las organizaciones requiere de una teoría del conocimiento dentro de las organizaciones donde la piedra angular precisamente es 
la distinción previamente realizada entre conocimiento tácito y conocimiento explícito. De este modo, el conocimiento es creado por los individuos, por lo que el conocimiento organizacional no es posible sin la colaboración de éstos últimos. El modelo de Nonaka y Takeuchi (1995) se contextualiza en un entorno social, donde se produce la conversión de unos tipos de conocimiento en otros, siendo necesario el proceso de interacción. Siguiendo a Nonaka y Takeuchi (1995) existen cuatro formas para la conversión del conocimiento: 1 . de tácito a tácito que llaman socialización, 2 de tácito a explícito o exteriorización, 3. de explícito a explícito o combinación y 4 . de explícito a tácito o interiorización.

Salazar y Zarandona (2007) plantean que existen otros modelos sobre la gestión del conocimiento en las organizaciones que a continuación se enuncian. El primero de ellos es el modelo de Wigg (1988, citado en Salazar y Zarandona, 2007), en el cual se engloba el proceso de creación, codificación y aplicación del conocimiento a la resolución de problemas en el que se identifica que las personas, a partir de una colección de información, determinan qué es necesario y qué no, analizando el tipo de conocimiento que tiene utilidad y cuál no. Por otro lado, el proceso de compartir el conocimiento es la base de la generación de nuevo conocimiento y es un medio para medir la eficiencia del sistema, al conformar repositorios de conocimiento relevante para la organización.

El modelo de Hedlund y Nonaka (1993, citado en Salazar y Zarandona, 2007) profundiza en los procesos de almacenamiento, transferencia y transformación del conocimiento. En el primero, se diferencia la articulación o conversión del conocimiento tácito en conocimiento explícito, y la internalización que convierte el conocimiento articulado en conocimiento tácito, en este proceso se utiliza la reflexión como un instrumento que permite el tránsito entre ambos tipos de conocimiento. El proceso de transferencia implica la transferencia de conocimiento desde el individuo a la organización y la apropiación o transferencia de conocimiento de la organización al individuo. El instrumento que posibilita tal proceso es el diálogo. Por último, la transformación importa y exporta conocimiento del entorno en cualquiera de sus formas, sea cognitivo, habilidades o conocimiento incorporado a la producción y servicio. 
Otro modelo es el de Grant (1996, citado en Salazar y Zarandona, 2007), éste posibilita la generación e integración de nuevo conocimiento. Considera que el conocimiento es individual y la organización tiene la tarea de coordinar, integrar y aplicar el conocimiento especializado de los miembros para la producción de bienes o servicios. El modelo propone cuatro mecanismos para integrar el conocimiento de la empresa. El primero consiste en materializar las reglas en procedimientos para desarrollar una tarea. El segundo es el diseño de las actividades como secuencias en el tiempo. El tercero consiste en generar rutinas organizativas, donde se indican las tareas a efectuar y la forma de llevarlas a cabo. En cuarto lugar, se encuentra la formación de grupos de resolución de problemas, que se implementan en el caso de tareas no rutinarias y complejas.

Por último, otro modelo ampliamente diseminado es el de KPMG Consulting (citado en Salazar y Zarandona, 2007), en el que se propone incrementar la capacidad de aprendizaje en las organizaciones, lo cual depende del compromiso de un equipo directivo con una visión organizativa que articule el aprendizaje en todos los niveles: personas, equipos y organización. Requiere también de la implementación de mecanismos para crear, captar, almacenar, transmitir y utilizar el conocimiento. Se encuentran involucrados seis elementos que permiten orientar el aprendizaje: estrategia, estructura organizativa, liderazgo, gestión de personas, sistemas de información, comunicación y cultural. Este modelo guarda semejanzas con el de Andersen (1998; 1999, citado en Salazar y Zarandona, 2007), el cual pretende acelerar el flujo de información entre los individuos y la organización y crear una infraestructura para conseguir una cultura orientada al aprendizaje y la innovación. 


\section{Tabla 1. Comparación entre distintos modelos de gestión del conocimiento organizacional}

\begin{tabular}{|c|c|c|c|c|}
\hline Modelo & Objetivo & Aportaciones & Medios & Limitaciones \\
\hline $\begin{array}{l}\text { Nonaka y } \\
\text { Takeuchi } \\
(1995)\end{array}$ & $\begin{array}{l}\text { Propiciar una } \\
\text { espiral de cono- } \\
\text { cimiento a tra- } \\
\text { vés del cual se } \\
\text { puede generar y } \\
\text { promover. }\end{array}$ & $\begin{array}{l}\text { Distinguir entre } \\
\text { dos tipos de } \\
\text { conocimiento tá- } \\
\text { cito y explícito. } \\
\text { En la dimensión } \\
\text { ontológica. }\end{array}$ & $\begin{array}{l}\text { Cuatro modos de } \\
\text { conversión del } \\
\text { conocimiento. } \\
\text { Socialización, } \\
\text { externalización, } \\
\text { combinación e } \\
\text { internalización. } \\
\text { Cinco fases del } \\
\text { modelo para } \\
\text { compartir conoci- } \\
\text { miento. }\end{array}$ & $\begin{array}{l}\text { No profundiza } \\
\text { en el proceso de } \\
\text { transferencia de } \\
\text { conocimiento im- } \\
\text { plícito a explícito. }\end{array}$ \\
\hline $\begin{array}{l}\text { Wiig } \\
(1988)\end{array}$ & $\begin{array}{l}\text { Reforzar el uso } \\
\text { del conocimien- } \\
\text { to. }\end{array}$ & $\begin{array}{l}\text { Describe el } \\
\text { contenido del } \\
\text { conocimiento, } \\
\text { su localización, } \\
\text { su proceso de } \\
\text { recolección, su } \\
\text { distribución y su } \\
\text { utilización. }\end{array}$ & $\begin{array}{l}\text { Recoger, forma- } \\
\text { lizar y codificar } \\
\text { el conocimiento. } \\
\text { Crear una estruc- } \\
\text { tura organizativa } \\
\text { del conocimiento. }\end{array}$ & $\begin{array}{l}\text { No distingue en- } \\
\text { tre la dimensión } \\
\text { epistemológica } \\
\text { y la dimensión } \\
\text { epistemológica. }\end{array}$ \\
\hline $\begin{array}{l}\text { Hedlund } \\
\text { y Nonaka } \\
\text { (1993) }\end{array}$ & $\begin{array}{l}\text { Almacenar, } \\
\text { transferir, } \\
\text { transformar el } \\
\text { conocimiento. }\end{array}$ & $\begin{array}{l}\text { Distingue entre } \\
\text { dos tipos de } \\
\text { conocimiento tá- } \\
\text { cito y articulado. }\end{array}$ & $\begin{array}{l}\text { Articulación e } \\
\text { internalización. } \\
\text { Extensión y } \\
\text { apropiación. } \\
\text { Asimilación y } \\
\text { diseminación. }\end{array}$ & $\begin{array}{l}\text { No profundiza } \\
\text { suficientemente } \\
\text { en la creación del } \\
\text { conocimiento. }\end{array}$ \\
\hline $\begin{array}{l}\text { Grant } \\
(1996)\end{array}$ & $\begin{array}{l}\text { Eficiencia de la } \\
\text { integración del } \\
\text { conocimiento } \\
\text { individual. }\end{array}$ & $\begin{array}{l}\text { Considera el } \\
\text { conocimiento } \\
\text { organizativo } \\
\text { como una } \\
\text { integración del } \\
\text { conocimiento } \\
\text { individual. }\end{array}$ & $\begin{array}{l}\text { Reglas materiali- } \\
\text { zadas en procedi- } \\
\text { mientos. Diseño } \\
\text { de actividades } \\
\text { productivas como } \\
\text { secuenciadas en } \\
\text { el tiempo. Rutinas } \\
\text { organizativas. } \\
\text { Formación de gru- } \\
\text { pos de resolución } \\
\text { de problemas. }\end{array}$ & $\begin{array}{l}\text { No reconoce la } \\
\text { existencia de un } \\
\text { conocimiento } \\
\text { organizativo de } \\
\text { naturaleza distinta } \\
\text { al conocimiento } \\
\text { individual. }\end{array}$ \\
\hline
\end{tabular}




\begin{tabular}{|c|c|c|c|c|}
\hline $\begin{array}{l}\text { KPMG } \\
\text { Andersen } \\
(1998, \\
1999)\end{array}$ & $\begin{array}{l}\text { Incrementar la } \\
\text { capacidad de } \\
\text { aprendizaje de } \\
\text { las organizacio- } \\
\text { nes. Conseguir } \\
\text { una cultura } \\
\text { organizativa } \\
\text { orientada al } \\
\text { aprendizaje. }\end{array}$ & $\begin{array}{l}\text { Resaltar los } \\
\text { elementos cultu- } \\
\text { rales. Aplicación } \\
\text { práctica en } \\
\text { empresas. }\end{array}$ & $\begin{array}{l}\text { Aprendizaje en } \\
\text { todos los niveles, } \\
\text { personas, equipos } \\
\text { y organización. } \\
\text { Infraestructura } \\
\text { organizativa, } \\
\text { estrategia, es- } \\
\text { tructura, lideraz- } \\
\text { go, gestión de } \\
\text { personas, sistemas } \\
\text { de información y } \\
\text { comunicación y } \\
\text { cultura. }\end{array}$ & $\begin{array}{l}\text { No distinguen } \\
\text { la dimensión } \\
\text { epistemológica } \\
\text { y la dimensión } \\
\text { ontológica. }\end{array}$ \\
\hline
\end{tabular}

Fuente: Elaboración propia con base en Salazar y Zarandona (2007).

En este caso se retoma el planteamiento tradicional de Nonaka y Takeuchi (1995), porque permite incorporando a los sistemas de la información y su constitución técnica y social, previendo que es en ésta última donde surge el aprendizaje organizacional, precisamente en la etapa de socialización. Además, se inicia la espiral de conocimiento con la exteriorización, dado que en esta forma es donde el conocimiento tácito pasa a ser explícito, visto no de manera informal, sino como estratégica del directivo para gestionar el conocimiento mediante los SI.

\subsection{Exteriorización: la gestión del conocimiento organizacional}

La exteriorización es el proceso en el cual el conocimiento tácito se vuelve explícito (Ver Tabla 1). En esta forma de conversión de conocimiento se provee el contexto adecuado para facilitar la creación y acumulación de conocimiento, desde el nivel individual hasta en comunidades de interacción cada vez mayores (Nonaka y Takeuchi, 1995). En este sentido, la gestión del conocimiento está vinculada a la selección de las estrategias organizacionales, es decir, el conjunto de compromisos que definen y racionalizan los objetivos y los métodos para alcanzarlos (Rivera, 2006). Desde esta óptica, la iniciativa se centra en el ápice estratégico de quien tiene la tarea de orientar la creación de conocimiento de los participantes (Nonaka, 2007). De este modo, la 
organización no solo procesa información del exterior al interior para adaptarse a los cambios, sino que crean nuevo conocimiento para redefinir los problemas y las soluciones (Nonaka y Takeuchi, 1995).

Las organizaciones que utilizan esta forma son concebidas como estratégicas, donde el conocimiento individual se convierte en organizacional al momento en que se crean, modifican o reemplazan las rutinas existentes mediante las cuales las organizaciones se construyen y operan (Alcover y Gil, 2002). La estrategia es la base de la organización y consiste en desarrollar la capacidad necesaria para adquirir, crear, acumular y explotar el conocimiento (Mintzberg, Ahlstrand y Lampel, 1999; Ramírez, Vargas y De la Rosa, 2011).

El estratega es el tomador de decisiones (Mintzberg, Ahlstrand y Lampel, 1999), las cuales son la base de la estrategia y contribuyen a dirigir el rumbo de la organización (Ramírez, Vargas y De la Rosa, 2011), no sólo en términos de la asignación de recursos de manera óptima para lograr los fines organizacionales (Arellano, 2008; Lovera, 2009), sino también para desarrollar la capacidad de gestionar el conocimiento (Andrade, 2003; Nonaka y Takeuchi, 1995).

La gestión del conocimiento se hace posible mediante una cultura sólida que afirme la estrategia (Nonaka y Takeuchi, 1995; Rivera, 2006). La cultura, siguiendo a Smircich (1983), es una noción que se retoma de la antropología para comprender los aspectos simbólicos de las organizaciones. Es un sistema de símbolos y significados compartidos que requieren ser interpretados para que puedan entenderse. Si la cultura está relacionada con el ápice estratégico se entiende que se habla de una cultura corporativa, emprendida por el gestor de la organización para legitimar el uso del SI y facilitar la labor de gestión del conocimiento. 
Tabla 2. Exteriorización

\begin{tabular}{|l|l|l|}
\hline $\begin{array}{l}\text { Forma de conversión } \\
\text { del conocimiento }\end{array}$ & \multicolumn{1}{|c|}{ Concepto } & \multicolumn{1}{|c|}{ Significado } \\
\hline Exteriorización & Tipo de conocimiento & $\begin{array}{l}\text { Conocimiento de tácito a explícito } \\
\text { (Nonaka y Takeuchi, 1995) }\end{array}$ \\
\cline { 2 - 3 } & Nivel de conocimiento & Individual a organizacional \\
\cline { 2 - 3 } & $\begin{array}{l}\text { Elementos que lo } \\
\text { posibilitan }\end{array}$ & $\begin{array}{l}\text { Estrategia Toma de decisiones } \\
\text { Cultura corporativa Metáforas } \\
\text { Discursos }\end{array}$ \\
\cline { 2 - 3 } & $\begin{array}{l}\text { Concepción de la } \\
\text { organización }\end{array}$ & Organización estratégica \\
\hline
\end{tabular}

Fuente: Elaboración propia.

La cultura corporativa es producida y administrada a través de dispositivos como valores, héroes, rituales y $\operatorname{artefactos}^{4}$, utilizados en función de conseguir eficacia organizacional y otros objetivos como supervivencia, generación de compromiso y sentido para moldear la conducta de los miembros (Deal y Kennedy, 1985; Shein, 2004). En este espacio se incluyen a las metáforas, las que para Nonaka y Takeuchi (1995) permiten que el conocimiento tácito se convierta en explícito al ratificar la creación de conceptos que puedan generar un modelo lógico, es decir un SI. Incluso, el ápice estratégico se vale de los recursos lingüísticos para involucrarse en la construcción de significados en el contexto de la interacción social cotidiana transmitiendo ideología, cultura y conocimiento (Alvesson y Kärreman, 2011; Fairclough, 1989). El discur-

4 Los valores conjuntan las creencias y los conceptos básicos de la organización, determinando normas de realización que están ligados al concepto de negocio. Por otra parte, los héroes son individuos que personifican los valores de la cultura y adecúan un modelo a seguir para encaminar la conducta y las actividades de los individuos en las organizaciones. Los rituales son rutinas programadas y sistemáticas de la vida cotidiana que muestran a los individuos el comportamiento que se espera de ellos, así los individuos no pierden tiempo en pensar cómo van a hacer las cosas o cómo deben reaccionar, generando con ello productividad. Los artefactos son productos visibles del grupo como estructuras, procesos, tecnologías e incluso creaciones artísticas que tienen lugar en las organizaciones (Deal y Kennedy, 1985; Shein, 2004). 
so les ayuda a legitimar en forma directa los procesos, aplicaciones y utilización de tecnologías permitiendo modelar la conducta y generar significados esperados.

\subsection{Combinación: los Sistemas de Información}

En términos de lo esgrimido por Nonaka y Takeuchi (1995), la combinación es un proceso de sistematización de conceptos con el que se genera un sistema de conocimiento (Ver Tabla 2). Esta forma de conversión requiere de la unión de distintos tipos de conocimiento explícito. Los individuos intercambian y combinan conocimiento a través de diferentes medios, el cual se clasifica, añade, combina y categoriza en nuevas formas. La combinación se lleva a cabo mediante los SI para facilitar la generación de conocimiento sistémico apoyado por las nuevas tecnologías y se integran a la estructura de la organización porque contribuyen al manejo de los recursos brindando posibilidades de enlace y conectividad. La tecnología, de acuerdo con el diseño de la estructura organizacional, involucra a las máquinas o equipos utilizados en el proceso productivo (Hall, 1983). El elemento tecnológico forma parte del orden técnico que apoya la estructura organizacional existente y posibilita la gestión y transferencia del conocimiento (Andrade, 2003), que en este caso lo conforman los SI.

La estructura se compone de las prácticas y procedimientos institucionalizados en la sociedad que las organizaciones incorporan a manera de mitos racionalizados (Meyer y Rowan, 1977). Está diseñada para administrar las actividades, la existencia de un orden jerárquico, la distribución de tareas, asignación de recursos, establecimiento de líneas de autoridad y comunicación para alcanzar los objetivos (Mazzotti, Gonzáles y Solís, 2011). Las funciones de ésta misma, según Hall (1983), son: producir resultados y alcanzar los objetivos organizacionales, minimizar la influencia de los individuos en la organización, ser el medio para tomar decisiones y llevar a cabo las actividades de las organizaciones.

La estructura tiene un impacto sobre los individuos, ya que de ellos depende la capacidad para innovar, lo cual, como se señaló previamen- 
te, es una característica crucial para la supervivencia de las organizaciones. Nonaka y Takeuchi (1995) recomiendan que el diseño de la estructura sea plano y flexible, garantizando en todo momento que las unidades estén interconectadas a una red de información.

Dado que los SI son el mecanismo para procesar la información de los individuos (Alcover y Gil, 2002), requieren de una definición formal de los procesos/prácticas para alimentar el software. Es por lo que, en ocasiones, la introducción de SI en la organización, conlleva al rediseño e incluso la depuración de procesos para garantizar la entrada de datos, su procesamiento y emisión de resultados de salida (Arellano, 2008). Los SI están involucrados con las áreas funcionales, en términos administrativos, para incrementar la eficiencia y la eficacia operativa (Abrego, Sánchez y Medina, 2016). La representación de ello está en la metáfora de organización como sistema, donde cada área tienen la capacidad de funcionar como un sistema más pequeño capaz de funcionar de manera independiente, pero interconectado (Abrego, Sánchez y Medina, 2016; Torres y Lamenta, 2015).

\section{Tabla 3. Combinación}

\begin{tabular}{|l|l|l|}
\hline $\begin{array}{c}\text { Forma de conversión } \\
\text { del conocimiento }\end{array}$ & \multicolumn{1}{|c|}{ Concepto } & \multicolumn{1}{c|}{ Significado } \\
\hline \multirow{4}{*}{ Combinación } & Tipo de conocimiento & $\begin{array}{l}\text { Conocimiento explícito } \\
\text { (Nonaka y Takeuchi, 1995) }\end{array}$ \\
\cline { 2 - 3 } & Nivel de conocimiento & Nivel técnico o estructural \\
\cline { 2 - 3 } & $\begin{array}{l}\text { Elementos que lo } \\
\text { posibilitan } \\
\text { Prácticas }\end{array}$ & Sistemas de Información \\
\cline { 2 - 3 } & $\begin{array}{l}\text { Concepción de la orga- } \\
\text { nización }\end{array}$ & $\begin{array}{l}\text { Organización como } \\
\text { sistema }\end{array}$ \\
\hline
\end{tabular}

Fuente: Elaboración propia.

\subsection{Interiorización: rutinas}

La interiorización es un proceso de conversión de conocimiento explícito a tácito, relacionada con el aprendizaje sobre la práctica. Cuando las experiencias son internalizadas en la base de conocimiento tácito 
de los individuos se vuelven valiosas. Para que se dé la creación de conocimiento organizacional es necesario que el conocimiento tácito acumulado en el plano individual se socialice con otros miembros y dar inicio a una espiral de creación de conocimiento. Se apoya de materiales como documentos, manuales o historias para ser presentado a los integrantes. Cuando el conocimiento explícito se convierte en tácito y es compartido por los miembros de la organización, se vuelve parte de la cultura organizacional (Nonaka y Takeuchi, 1995).

La interacción con la estructura en el marco de las actividades y el uso de SI se normaliza mediante rutinas, es decir, actividades laborales diarias basadas en procedimientos asentados en normas y reglamentos (Lovera, 2011). Las rutinas tienen expresión en el proceso de transformación del conocimiento explícito a tácito. Son la llave a partir de la cual las organizaciones mantienen la consistencia y almacenan el conocimiento organizacional, constituyendo una especie de memoria organizacional, de modo que el conocimiento sobre cómo realizar tareas se almacena colectivamente (Reay y Golden-Biddle, 2003).

Las rutinas consisten en patrones repetitivos de acciones interdependientes, pero no estáticas. Pueden documentarse en un conjunto de procedimientos formales o reglas, de modo que funcionen como tipo ideal, pero en la práctica consisten en acciones, lugares y momentos específicos, por lo que son flexibles al permitir incorporar cambios, es decir, nuevos aspectos prácticos a la rutina (Fieldman y Pentland, 2003).

Para posibilitar la interiorización del conocimiento explícito a tácito es fundamental el aprendizaje organizacional ${ }^{5}$. En un primer momento el aprendizaje individual, siendo la base del conocimiento organizacional, se codifica en las rutinas. Posteriormente, el aprendizaje grupal servirá para socializar el conocimiento en la siguiente forma al distribuirse

5 Garzón y Fisher (2008) explican que el término se asocia a la gestión del conocimiento bajo el argumento de que, si se comprenden mejor los procesos claves y las situaciones específicas, se aprovecha la experiencia y el conocimiento acumulado grupalmente en beneficio de los miembros de la organización y la consecución de los objetivos de ésta última. 
en la organización a nivel colectivo (Alcocer y Gil, 2002). Lo anterior, permite denominar a la organización en esta manera de conversión de conocimiento como organización que aprende. Una organización que aprende es aquella donde los miembros adquieren, comparten y combinan conocimiento teniendo como resultado un proceso colectivo de trabajo conjunto en que se aprende a compartir información y crear conocimiento (Alcover y Gil, 2002; Garzón y Fisher, 2008). Este tipo de organización se caracteriza por ser descentralizada, estimular la comunicación y alentar a los individuos al trabajo en equipo (Mintzberg, Ahlstrand y Lampel, 1999).

\section{Tabla 4. Interiorización}

\begin{tabular}{|l|l|l|}
\hline $\begin{array}{c}\text { Forma de conversión } \\
\text { del conocimiento }\end{array}$ & \multicolumn{1}{|c|}{ Concepto } & \multicolumn{1}{c|}{ Significado } \\
\hline \multirow{4}{*}{ Interiorización } & Tipo de conocimiento & $\begin{array}{l}\text { Conocimiento de explícito } \\
\text { a tácito (Nonaka y Takeu- } \\
\text { chi, 1995) }\end{array}$ \\
\cline { 2 - 3 } & Nivel de conocimiento & Técnico a individual/ social \\
\cline { 2 - 3 } & $\begin{array}{l}\text { Elementos que lo } \\
\text { posibilitan }\end{array}$ & $\begin{array}{l}\text { Aprendizaje organizacional } \\
\text { Rutinas }\end{array}$ \\
\cline { 2 - 3 } & $\begin{array}{l}\text { Concepción de la } \\
\text { organización }\end{array}$ & Organización que aprende \\
\hline
\end{tabular}

Fuente: Elaboración propia.

\subsection{Socialización: creación del conocimiento}

La socialización para Nonaka y Takeuchi (1995) consiste en compartir experiencias y crear conocimiento tácito. Los individuos pueden adquirir este tipo de conocimiento en el uso del lenguaje, observación, imitación y práctica, a través de la socialización para compartir el conocimiento tácito. En esta forma de conversión del conocimiento, se promueve la interacción de los miembros al compartir sus experiencias fomentando la mayor autonomía posible. Como intuyen los autores, la cultura corporativa y el pensamiento estratégico del ápice son fundamentales para encaminar el comportamiento de los individuos, no obstante, en este punto, se involucra la cultura organizacional, producto de los grupos informales de miembros que están en contacto con la 
realidad de la organización e interpretan el conocimiento explícito para construir sus propios significados en situaciones cotidianas (Arellano, 2008; Nonaka, 2007).

La cultura organizacional discrepa de la cultura corporativa, en el sentido de que ésta última tiene una visión mecanicista o encaminada a legitimar las actividades a través de ideologías cultivadas por el ápice estratégico (Smircich, 1983). Por el contrario, la cultura organizacional refiere al sentido que generan los participantes de una organización en el marco de su vida cotidiana. Su origen está en los grupos informales de la organización y los significados que producen al comprender valores y normas desarrollados en su experiencia e interactuar con la estructura organizacional (Barba y Solís, 1997; Montaño, 2003). Para la conversión del conocimiento de tácito a tácito se requiere de la socialización, la cual acontece en el marco de la cultura organizacional.

El desarrollo de los SI permite socializar la información en redes de conocimiento no sólo de manera interna en cada subsistema, sino que, dada la necesidad de interconexión entre organizaciones, pensando en un uso estratégico, por ejemplo, contribuye a reducir costos de operación, generar estrategias conjuntas, facilitar fusiones, mejorar la relación entre proveedores, entre otros (Abrego, Sánchez y Medina, 2016). La información externa es vital para la toma de decisiones ya que permanece en constante cambio (Arellano, 2008). De forma general, las redes permiten un manejo eficiente de la producción, transferencia y transmisión de los conocimientos, teniendo potencial para alentar la creatividad de los miembros y convertirla en innovación. Una red de conocimientos permanece mientras exista la colaboración entre los miembros y se desarrollen actividades flexibles y con diferentes niveles de realización e impacto (Mazzotti, Gonzáles y Solís, 2011).

Como producto de la implantación de un SI las organizaciones logran innovaciones importantes, ya que éstos llegan a desempeñar un papel determinante que garantiza resultados permanentes (López, 2002; Lovera, 2009) (Ver Tabla 4). Lo cual es producto de la interacción en la 
construcción de conocimiento e irrumpe en las rutinas organizacionales (Mazzotti, Gonzáles y Solís, 2011). La flexibilidad estructural y el constate proceso de creación de conocimientos fomenta la innovación constante, de este modo las organizaciones se erigen como innovadoras.

\section{Tabla 5. Socialización}

\begin{tabular}{|l|l|l|}
\hline $\begin{array}{c}\text { Forma de conversión } \\
\text { del conocimiento }\end{array}$ & \multicolumn{1}{|c|}{ Concepto } & \multicolumn{1}{c|}{ Significado } \\
\hline \multirow{5}{*}{ Socialización } & Tipo de conocimiento & $\begin{array}{l}\text { Conocimiento tácito a tácito } \\
\text { (Nonaka y Takeuchi, 1995) }\end{array}$ \\
\cline { 2 - 3 } & Nivel de conocimiento & $\begin{array}{l}\text { Individual, grupal, organiza- } \\
\text { cional, inter organizacional }\end{array}$ \\
\cline { 2 - 3 } & $\begin{array}{l}\text { Elementos que lo } \\
\text { posibilitan }\end{array}$ & $\begin{array}{l}\text { Cultura organizacional } \\
\text { Lenguaje, observación, imi- } \\
\text { tación y práctica (Nonaka y } \\
\text { Takeuchi, 1995) }\end{array}$ \\
\cline { 2 - 3 } & $\begin{array}{l}\text { Concepción de la } \\
\text { organización }\end{array}$ & Organización innovadora \\
\hline
\end{tabular}

Fuente: Elaboración propia.

\section{Conclusiones}

Como se ha podido apreciar a lo largo del texto, los sistemas de la información juegan un papel relevante en la gestión del conocimiento organizacional. Son herramientas que apoyan en la extracción, procesamiento, almacenaje y distribución de la información. En primer lugar, tienen el propósito de integrar el proceso y recuperación de información para su utilización estratégica. Autores como Alcover y Gil (2002) les califican como repositorios del conocimiento y las tareas de los miembros, o sistemas de memoria grupal y organizacional.

En segundo lugar, los sistemas de la información posibilitan la transición entre el conocimiento organizacional e individual, ya que la gestión del conocimiento requiere que la información se distribuya a través del sistema, los SI permiten que se codifique en un modelo que, ante 
su utilización, es transmitido a los individuos institucionalizándose en rutinas que posteriormente son difundidas en los procesos de socialización, educación, profesionalización, imitación e intercambio (Alcover y Gil, 2002) para nuevamente generar conocimiento. En su carácter de instrumentos de apoyo en el procesamiento de la información permiten construir esquemas de aprendizaje colectivo.

En tercer término, los sistemas de la información tienen preponderancia en la forma de conversión del conocimiento denominada por Nonaka y Takeuchi (1995) como combinación, en la cual ocurre un proceso de sistematización de conceptos para generar un sistema de conocimiento. La combinación se lleva a cabo mediante los SI, facilitando la generación de conocimiento sistémico apoyado por las TIC e integrándose a la estructura de la organización brindando posibilidades de enlace y conectividad. En la forma de exteriorización, el conocimiento tácito se convierte en explícito ante la iniciativa del ápice estratégico de favorecer las condiciones para crear y gestionar el conocimiento, tal decisión se apoya en el elemento tecnológico que proveen los SI, pero también implica el fortalecimiento de una cultura corporativa que permita legitimar los procesos, aplicaciones y utilización de las tecnologías. En la interiorización el conocimiento explícito contenido en los SI se convierte en tácito al momento de transfigurarse en una rutina, ésta última consiste en patrones repetitivos de acciones basadas en procedimientos asentados en reglas formales que se derivan de la estructura. En esta esfera es primordial el aprendizaje individual, siendo la base del conocimiento organizacional. Por último, en la socialización se comparten experiencias y se crea conocimiento tácito. La interacción se da en grupos informales donde se comparten significados y se interpreta la realidad organizacional, generando nuevos conocimientos y, por ende, innovación.

Finalmente, cada organización crea sus propias estrategias, estructuras y forma de gestionar el conocimiento a partir de los Sistemas de Información. La aproximación que se ha presentado tiene un carácter descriptivo. Quedaría pendiente llevar este desarrollo a la investigación de campo mediante un estudio de caso que permita analizar las particularidades acerca de cómo una organización pública o privada gestiona el conocimiento a partir de SI. 


\section{Bibliografía}

Abrego, A.D., Sánchez, T. Y. y Medina, Q. J. M. “Influencia de los sistemas de información en los resultados organizacionales". Contaduría y Administración, 62, Universidad Nacional Autónoma de México (UNAM): México, 2016, pp. 303-320.

Alcover, C. M. y Gil, F. "Crear conocimiento colectivamente: aprendizaje organizacional y grupal”. Revista de Psicología del Trabajo y de las Organizaciones, 18(2-3), Universidad Rey Juan Carlos: Madrid, España, 2002, pp. 259-301.

Álvarez, M. M. R. "Procesamiento y gestión digital de la información". Biblioteca Universitaria. 9(2), Universidad Nacional Autónoma de México (UNAM): México, 2006, pp. 144-151.

Alvesson, M. \& Kärreman D., "Decolonializing discourse: Critical reflections on organizational discourse", Human Relations, 64(9), SAGE Journals, The Tavistock Institute, 2011, pp. 1121-1146.

Andrade C. J. A. "Tecnologías y sistemas de información en la gestión del conocimiento en las organizaciones". Revista Venezolana de Gerencia, 8(24), Universidad de Zulia, Venezuela, 2003, pp. 558-574.

Arellano, R. M. "Sistemas de información: ¿adecuación a los cambios tecnológicos o herramienta de gestión?". Revista de Ciencias Sociales, 14(3), Revista de Ciencias Sociales, Maracaibo, 2008, pp. 528-545.

Barba, A.A. \& Solís P.C. Cultura en las organizaciones Enfoques y Metáforas de los Estudios Organizacionales. Vertiente Editorial, México, 1997. Disponible en: https://uami.wikispaces.com/Cultura+en+las+organizaciones. +Enfoques $+y+$ Metaforas + de + los + Estudios+Organizacionales.

Castells, M. La era de la información: economía, sociedad y cultura. Volumen I. La sociedad Red. Alianza Editorial, Madrid, 2000. p. 628 . 
Deal, T. E. \& Kennedy, A. A. Culturas corporativas, Ritos y rituales de la vida organizacional. Fondo Educativo Interamericano, México, 1985.

Didriksson, T. A. (2015). El futuro anterior. La universidad como sistema de producción de conocimientos, aprendizajes e innovación social. CLACSO. Disponible en: http://biblioteca.clacso.edu.ar/clacso/ becas/20150102084612/Ensayo.pdf, [Consultado el 27 de febrero de 2018].

Didriksson, T.A, "Economía del conocimiento y universidad pública en América Latina". Revista Latinoamericana de Educación Comparada, (10), Sociedad Argentina de Estudios Comparados en Educación, Buenos Aires, Argentina, 2016, pp. 21-34.

Fairclough, N. Language and Power. Longman Group Limited. New York, 1989. $259 \mathrm{p}$.

Feldman, M. S. y Pentland B. T. "Reconceptualizing Organizational Routines as a Source of Flexibility and Change". Administrative Science Quarterly, 48, SAGE Publications, Los Ángeles, 2003, pp. 94-118.

García, A. M. y Gómez V. M. "Prácticas de gestión del conocimiento en los grupos de investigación: estudio de un caso". Interamericana, 38(1), Biblioteca Interamericana, Medellín, 2014, pp. 13-25.

Garzón C. M. A. y Fisher, A. L. "Modelo teórico de aprendizaje organizacional". Pensamiento y Gestión, (24), Revista de la Escuela de negocios de la Universidad del Norte, 2008, pp. 195-224.

Gil, M. M. D., López, O. G., Molina, G. C. y Bolio, Y. C. “La gestión de la información como base de una iniciativa de gestión del conocimiento". Ingeniería Industrial, 23(3), Instituto Superior Politécnico José Antonio Echeverría, La Habana Cuba, 2011, pp. 231-237.

Hall, R. Organización, estructura y procesos. Editorial Dossat S. A. Madrid, España, 1983, 353 p. 
Kafure, I. "El proceso creativo de la interfaz del sistema de gestión de la información”. Revista Interamericana de Bibliotecología, 33(1), Universidad de Antioquía, Colombia, 2010, pp. 169-186.

Lindblom, A. y Tijjanen "Knowledge creation and business format franchising. Knowledge Management Practices in higher education”. Educause Quarterly, Estados Unidos (4), 2010, pp. 28-33.

López, G. M. P. "Los sistemas computarizados de información en las organizaciones y sus implicaciones éticas, sociales y humanas". Gestión y estrategia, (22), Universidad Autónoma Metropolitana Azcapotzalco, 2002, pp. 80-90.

Lovera, A. M. I. "La organización creadora de conocimiento: una perspectiva teórica”. Ominia, (2), Sistema de Servicios Bibliotecarios y de Información, Maracaibo Venezuela, 2009, pp. 178-193.

Lovera, A. M. I. "Rutinas organizativas académicas como impulsoras del desarrollo organizacional universitario". Omina, 17(2) Universidad de Zulia, Maracaibo Venezuela, 2011, pp. 103-112.

Mazzotti, P.G., González, C. R. Y Solís, P. P. C. (2011). "De la transmisión a la co-creación: cambio en el tipo de conocimiento y en las necesidades de formación profesional del administrador". XVI Congreso Internacional de Contaduría Administración e Informática. Disponible en: http: //congreso.investiga.fca.unam.mx/docs/xvi/ docs/9B.pdf, [Consultado el 27 de febrero de 2018].

Mena, E.N. "Firefox: una herramienta para la gestión de la información en las organizaciones". Ciencias de la Información, Cuba, 42(1), 2011, pp. 71-75.

Meyer, J. \& Rowan, B. "Institutionalized Organizations: Formal structure as myth and ceremony". The American Journal of Sociology, 83 (2), The University of Chicago Press Journals, Chicago, 1977, pp. $340-363$.

Meyer, J. "Conclusion: Institutionalization and the Rationality of Formal Organizational Structure". Organizational environments: Ritual and rationality, Beverly Hills: Sage. 1992, pp. 261-281. 
Mintzberg, H., Ahlstrand, B. y Lampel, J. Safari a la Estrategia. Una visita guiada por la jungla del management estratégico. Gránica, México, 1999. 512 p.

Montaño, H.L. "Modernidad y cultura en los Estudios Organizacionales. Tres modelos analíticos”. Iztapalapa, (55), Universidad Autónoma Metropolitana, Iztapalapa, 2003, pp. 15-33.

Montaño, H.L. "Organisational models and culture: a reflection from Latin America". European Journal of International Management, 3(2), Polytechnic Institute of Leiria, 2009, pp. 146-166.

Morales, L.V. "La transferencia de conocimiento en las organizaciones". Estudios Interdisciplinarios de la Organización, 1(1), Universidad de Guanajuato, 2012, pp. 50-65.

Moya-Angeler, J. “Origen y situación actual de la gestión del conocimiento". Economistas, (87), 2001, pp. 397-401.

Mumby, D. K. "The political Function of Narrative in Organizations". Communication Monographs, (54), National Communication Association, Washington DC 1987, pp.113-127.

Nonaka, I. (2007). "La empresa creadora de conocimiento". Harvard Business School Publishing Corporation. Recuperado de: https: // bschogardecristo.files.wordpress.com/2007/08/nonaka_red.pdf, [Consultado el: 27 de febrero de 2018].

Nonaka, I. y Takeuchi H. La organización creadora de conocimiento. Como las compañias japonesas crean la dinámica de la innovación. Oxford University Press, México, 1995.

Polanyi, M. Personal Knowledge. Towards a Post-Critical Philosophy. Routledge, London 1958, 493 p.

Ramírez, M. G., Vargas, L. G. y De la Rosa, A. A. "Estudios organizacionales y administración. Contrastes y complementariedades: Caminando hacia el Eslabón Perdido". Revista Forum Doctoral, (3), Red de Posgrados de Investigación Latinoamericanos en Adminis- 
tración y Estudios de la Organización, Medellín Colombia, 2011, pp.7-54.

Reay, T. y Golden-Biddle, K. Learning how to change organizational routines. Disponible en: https: //www2.warwick.ac.uk/fac/soc/wbs/ conf/olkc/archive/oklc4/papers/oklc2003_reay.pdf, [Consultado el: 27 de febrero de 2018].

Rivera, B. J. G. “¿Gestión del conocimiento o gestión de la información?”. Tecnológicas, (16), Instituto Tecnológico Metropolitano, Medellín, 2006, pp. 59-82.

Salazar, C. J. M. y Zarandona, A. X. "Valoración crítica de los modelos de gestión del conocimiento". Congreso Anual AEDEM, Universidad Rey Juan Carlos. Madrid. 2007, pp. 1-15. Disponible en: https:// dialnet.unirioja.es/servlet/articulo?codigo $=2527673$. [Consultado el: 29 de mayo de 2018].

Shein, E. Organizational culture and leadership, Jossey-Bass. San Francisco, 2004, p. 437.

Silva, M.R. "Los sistemas de información como arma estratégica en la gestión empresarial”. Perspectivas, Bussiness School Universidad de Navarra, Barcelona España, 9(18), Falta Editor y país, 2006, pp. 161-176.

Smircich, L. "Concepts of Culture and Organizational Analysis". Administrative Science Quarterly, 28(3), Sage, Beverly Hills, 1983, pp. 339-358.

Sveiby, K. E. "The intangible assets monitor". Journal of Human Resource Costing and Accounting, 2(1), Emerald Insight, West Yorkshire, 1997, pp. 73-97.

Tejedor, B. y Aguirre, A. "Proyecto Logos: investigación relativa a la capacidad de aprender de las empresas españolas". Boletín de Estudios Económicos, 53(164), Universidad Comercial de Deusto, Madrid España 1998, pp. 231-249. 
Torres, K. y Lamenta P. “La gestión del conocimiento y los sistemas de información en las organizaciones”. Negotium, 11(32), Fundación Unamuno, Venezuela, 2015, pp. 3-20.

Vessuri, H. "De la pertinencia social a la sociedad del conocimiento. En Tunnermann”, Ed. B. C., La educación superior en América Latina y el Caribe: diez años después de la Conferencia Mundial, pp. 459-477. Disponible en: http: //www.unesco.org.ve/dmdocuments/ biblioteca/libros/capitulos/ESD_1998/ESD_1998_CAP_9.pdf, [Consultado el: 27 de febrero de 2018]. 